\title{
Excision and Inter-Positional Sural Nerve Graft for Post-Traumatic Non-Conducting Ulnar Nerve Neuroma
}

\author{
AMR MOHAMED ELHUSSINY KHATER, M.D.*; REDA ABDALLAH YOUNIS, M.D.* and \\ NEHAL THARWAT MOHAMED EL-SAYED, M.D.** \\ The Departments of Plastic Surgery* and Radiodiagnosis**, Faculty of Medicine, Mansoura University
}

\begin{abstract}
Introduction: Ulnar nerve injuries around the wrist result in paralysis of intrinsic muscles and sensory loss of little finger and ulnar half of ring finger. Formation of traumatic neuroma of the ulnar nerve adds pain at the site of nerve repairs.

Patients and Methods: 10 patients were included in this study. Clinical, radiological and electrophysiological assessment indicate surgery in these patients where neuroma was excised, identification of sensory and motor fascicles of the ulnar nerve was done and cable nerve grafts were used to bridge the nerve defects. Assessment of the outcome was done in patients who finished follow-up for at least one year.

Results: Pain at the site of neuroma disappeared after surgery. All patients were followed-up and 6 patients needed tendon transfer after one year. Sensory recovery was achieved after one year. Trophic changes and cold intolerance was improved.
\end{abstract}

Discussion: Primary repair of ulnar nerve injuries is the gold standard treatment for such injuries. Claw hand, weakness of the hand grip and abduction deformities are the main motor defects which can be corrected by tendon transfers. However, sensory defects and cold intolerance affected the outcome of surgery. When the diagnosis of non-conducting neuroma is made, neuroma resection and reconstruction improve the clinical outcome.

Conclusion: The unfavorable outcomes of ulnar nerve injuries can be improved by resection of neuroma in continuity and nerve grafting. Multidisciplinary team is needed to achieve the best clinical outcomes.

Key Words: Ulnar nerve - Fascicular repair - Neuroma Wallerian degeneration.

\section{INTRODUCTION}

Traumatic neuroma results from abnormal regeneration of injured peripheral nerves secondary to stretch, pressure, nerve lacerations or crush injuries [1]. It is manifested by continuous pain, sensory abnormalities and tender mass at an anatomical site of a peripheral nerve [2]. In addition, improper repair of transected peripheral nerve can end with neuroma formation. Internal neurolysis, nerve undermining and dissections, damage to nutrient blood vessels and incorrect nerve suturing are predisposing factors to neuroma formation [3].

When the perineurium is destroyed, the regenerating axons escape into the surrounding tissues in an irregular pattern forming whorls, convolutions and spirals. Neuroma is formed mainly of disorganized nerve axons, Schwann cells, fibroblasts and blood vessels [4].

Ulnar nerve injuries in the distal forearm result in sensory loss in the ulnar half of ring finger and little finger. The main motor defects include clawing deformity of the hand, abduction deformity of the little finger and weak power grip. These defects results from paralysis of the ulnar nerve innervated intrinsic muscles of the hand [5].

Ulnar nerve injuries with or without median nerve injuries are the commonest peripheral nerve injuries [6]. The outcome of reconstruction of ulnar nerve injuries is inferior to other nerve injuries in the upper limb $[\mathbf{7 , 8}]$. This fact can be explained by the large motor component of the ulnar nerve, which is the main nerve supply to the intrinsic muscles of the hand. These muscles are difficult to be innervated after ulnar nerve injuries due to long time needed to complete nerve regeneration and muscle re-innervation [9].

In this article, we will evaluate the results after surgical correction of neuroma in continuity of the ulnar nerve using nerve grafting and fascicular nerve repair.

\section{Surgical anatomy of the ulnar nerve:}

The ulnar nerve emerges from the medial cord of the brachial plexus from the spinal segments of $\mathrm{C} 8$ and $\mathrm{T} 1$ [10]. It enters the forearm between the 
heads of the flexor carpi ulnaris (FCU). In the lower half of the forearm, it gives rise to dorsal cutaneous branch. Then, the main trunk of the ulnar nerve, runs between the ulnar vessels laterally and FCU tendon medially. Later, a palmar cutaneous branch is given off the main trunk. Within the Guyon's canal, it is divided into superficial sensory branch and deep motor branch [11].

The understanding of the fascicular pattern of the peripheral nerves is important for the success of peripheral nerve surgery. In the proximal parts of a peripheral nerve, there is funicular pattern of nerve fascicles where fascicular interconnection and crossings are common [12]. However, in the terminal branches of the peripheral nerves, there are well-formed separated fascicles with group arrangement [13]. Later, Williams and Jabaley document the presence of group arrangement of nerve fascicles with similar functions throughout the length of peripheral nerves [14].

Chow and his colleagues detect the presence of a well-formed motor fascicular group of the ulnar nerve up to $90 \mathrm{~mm}$ proximal to the radial styloid. This motor fascicle is located on either the dorsal or the ulnar-dorsal aspect of the ulnar nerve in the distal forearm [15]. However, at Guyon's canal, the motor fascicle passes radially to forms the deep branch of the ulnar nerve supplying the intrinsic muscles of the hand. The size proportions of the sensory and motor groups at this distal level is approximately $3: 2[\mathbf{1 6}]$.

\section{PATIENTS AND METHODS}

This study included 10 patients who had injury of the ulnar nerve and had an attempt of surgical repair since at least 1 year ago with no signs of nerve recovery. Informed consents were obtained from our patients. Patient's demographic data were shown in Table (1).

Table (1): Patient's demographic data.

\begin{tabular}{cccccc}
\hline & Age & $\begin{array}{c}\text { Time from previous } \\
\text { repairs in months }\end{array}$ & Sex & $\begin{array}{c}\text { Associate } \\
\text { injuries }\end{array}$ & $\begin{array}{c}\text { Length of } \\
\text { graft }\end{array}$ \\
\hline 1 & 26 & 15 & F & & 65 \\
2 & 49 & 18 & M & Ulnar vessels & 40 \\
3 & 19 & 13 & M & Ulvar vessels & 50 \\
4 & 25 & 22 & M & Flexor tendon & 40 \\
5 & 39 & 26 & M & & 40 \\
6 & 55 & 24 & M & Median N. & 50 \\
7 & 24 & 14 & M & & 48 \\
8 & 33 & 17 & F & & 55 \\
9 & 42 & 15 & F & Flexor tendon & 35 \\
10 & 35 & 18 & M & Ulnar vessels & 45 \\
\hline
\end{tabular}

\section{Inclusion criteria:}

1- Patients who complained of persistent localized pain and discomfort at the location of previous ulnar nerve injuries at the distal forearm.

2- Previous attempts of ulnar nerve repair more than 12 months.

3- No recovery of the ulnar nerve innervated intrinsic hand muscles and sensory loss of the little finger and medial half of ring finger.

\section{Exclusion criteria:}

1- Patient age less than 10 years or older than 60 years.

2- Patients with co-morbid diseases including renal impairments, advanced malignancy, diabetes mellitus and advanced cardiac diseases.

3- Patients unwilling to perform postoperative rehabilitations.

\section{Preoperative assessment:}

We obtained history of the nature of Injury, hand dominance, associated injuries and previous surgeries. Complete hand examination was done including full neurovascular examination. Plain $\mathrm{X}$-ray was done to rule out hand fractures and deformities. High-frequency ultrasound examination revealed localized swelling of the nerve while nerve continuity was maintained Fig. (1). Electromyogram (EMG) of the interossei muscles and hypothenar muscles revealed no peaks of compound muscle action potentials (CMAPs) in the affected muscles.

The diagnosis of ulnar nerve neuroma was done when there is history of ulnar nerve injury for more than 1 year complicated by painful tender swelling with distal sensory loss and muscle fibrillations recorded by EMG of ulnar nerve neurotized muscles. The diagnosis was confirmed with ultrasound examination.

\section{Surgical technique:}

Under general anesthesia, we placed the patient in the supine position with his affected upper limb abducted and placed over a side table. The patient was prepped and draped. Under tourniquet control, complete aseptic technique and loupe magnification, skin incision was done. We either performed surgical revision of previous scars or used zigzag incision. Sharp dissection was used to isolate the affected nerve as in Fig. (2). Intraoperative nerve stimulation was used to confirm the absence of distal muscle contractions secondary to proximal nerve stimulation. 
Excision of the affected nerve segment was done until the appearance of healthy fascicular pattern of the ulnar nerve proximally. Minimal interfascicular dissection and release interfascicular fibrosis were used to expose the distal end of the transected nerve. Release of the distal nerve segment was done by releasing the Guyon's canal. Sural nerve grafts were obtained from the leg as in Fig. (3) using multiple incisions on the outer aspect of the leg. The sural nerve was cut into multiple grafts.

By the use of operative microscope, Individual fascicular nerve grafting was done using eight zero nylon sutures with rounded tip needles. We started by two stay sutures using eight zero nylon and finish our repair using 10 zero nylon sutures. Later, tourniquet was deflated, hemostasis was achieved, Penrose drains was inserted, and wound closure was done. Below elbow splint was used to immobilize the limb Figs. $(4,5)$.

\section{Postoperative care and follow-up:}

Dressing changes, drains removal and patients were discharged on the first day after surgery. The splint was removed after 2 weeks and physiotherapy exercises was started from the third week. After one year, Motor and sensory assessment were done.

We tested adduction in small finger and abduction in index and small finger as assessment test for motor function and pain and 2-point discrimination test for grading the sensory outcome.

We used Medical Research Council scale. For sensory evaluation, we graded our patients as S0 when there was no sensation, S1 when there was recovery of deep cutaneous pain, $S 2$ when there was recovery of superficial cutaneous pain and some touch sensation, S3 when there was recovery of superficial cutaneous pain and touch sensation and S4 when there was normal sensation. For motor evaluation, we graded our patients as $\mathrm{M} 0$ when there was no contraction, M1 when there was flicker of muscle contraction, M2 muscle movement when gravity was eliminated, M3 when there was muscle movement against gravity, M4 when there was muscle movement against some resistance and M5 when there was normal muscle power [17].

\section{RESULTS}

Two patients were excluded from the study due to travelling outside the country and two patients were excluded due to poor compliance to prolonged physiotherapy and rehabilitation protocols. Six patients who finished the one year follow-up. One patients was females and five cases were males.
The mean time of follow-up period of our patients was 20.5 months (range from 15-26 months). Age ranged from $19-55$ years (mean 35.5 years). The mean time of surgery after the initial injury was 19.67 months (range frond m13-26 months). The length of nerve cable grafts ranged from 40 to 65 millimeters (mean 47.5 millimeters).

After at least one year of follow-up, evaluation of the outcome of surgery was done. In these patients, pain at the site of the neuroma was relieved. Sensory functions grades were $\mathrm{S} 3$ in four cases and S2 in two cases. Motor function grades were M3 in three cases, M2 in two cases and M1 in one case.

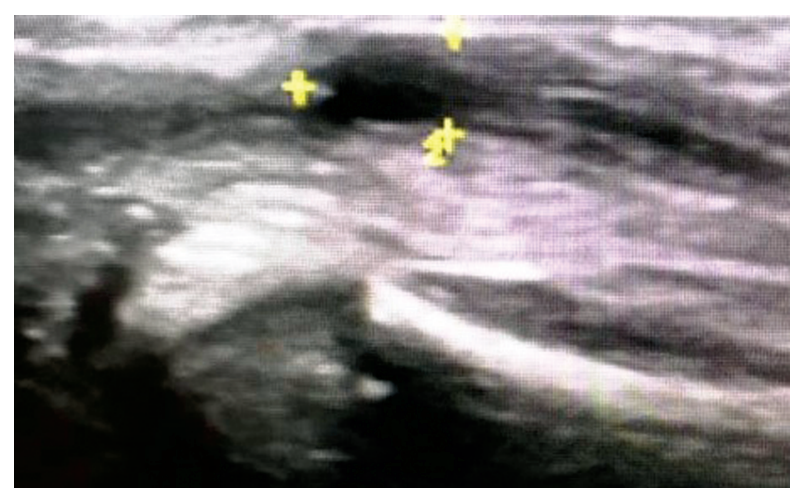

Fig. (1): Ultrasonic examination of the ulnar nerve showed localized thickening of the nerve (neuroma) as shown between yellow marks while nerve continuity was maintained.

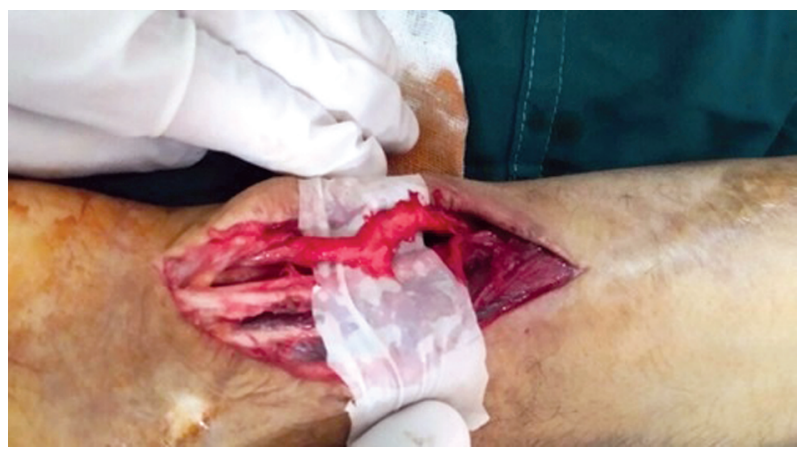

Fig. (2): Intraoperative photo of ulnar nerve neuroma in continuity.

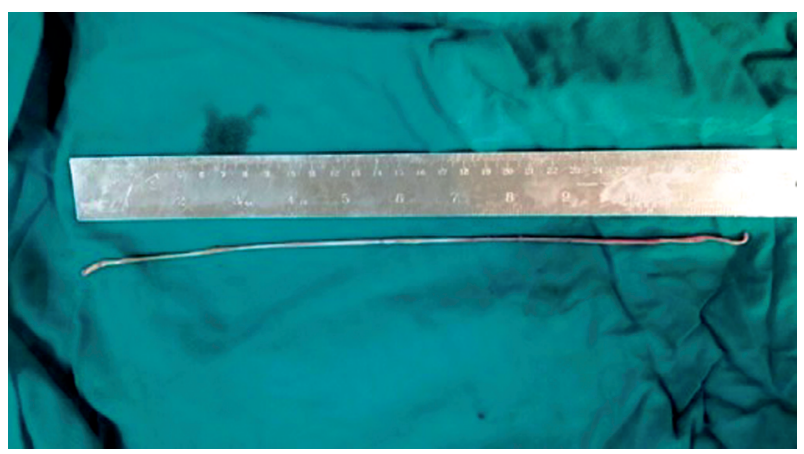

Fig. (3): Sural nerve grafts obtained as long as 30 centimeter. 

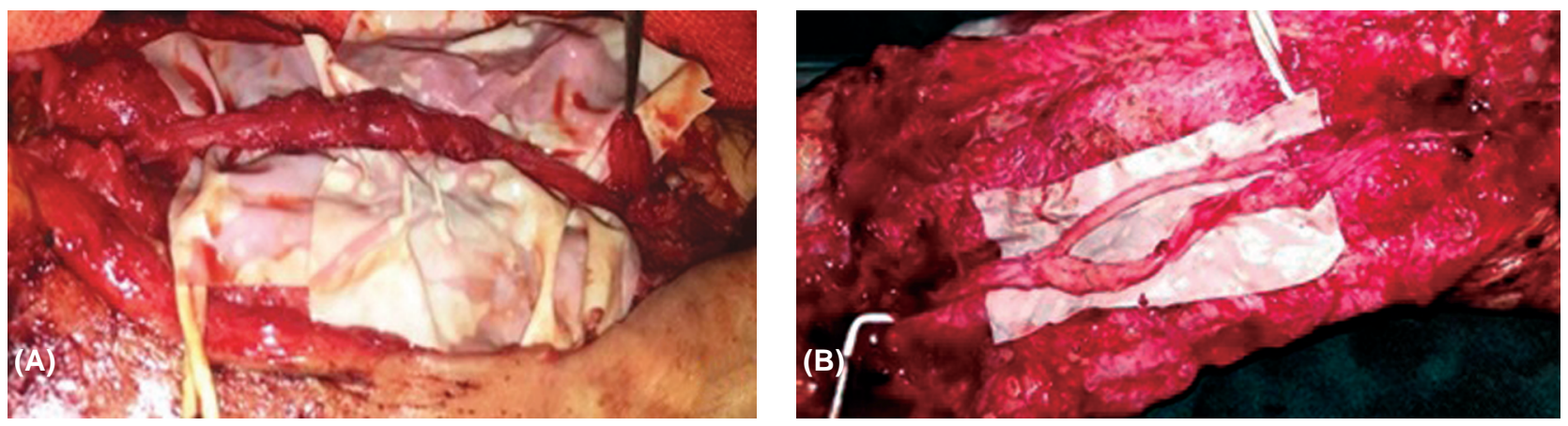

Fig. (4): (A): Intraoperative photo of ulnar nerve neuroma. Note that distal sensory fascicle was not repaired. (B): Individual fascicular repair using cable nerve grafts from sural nerve.
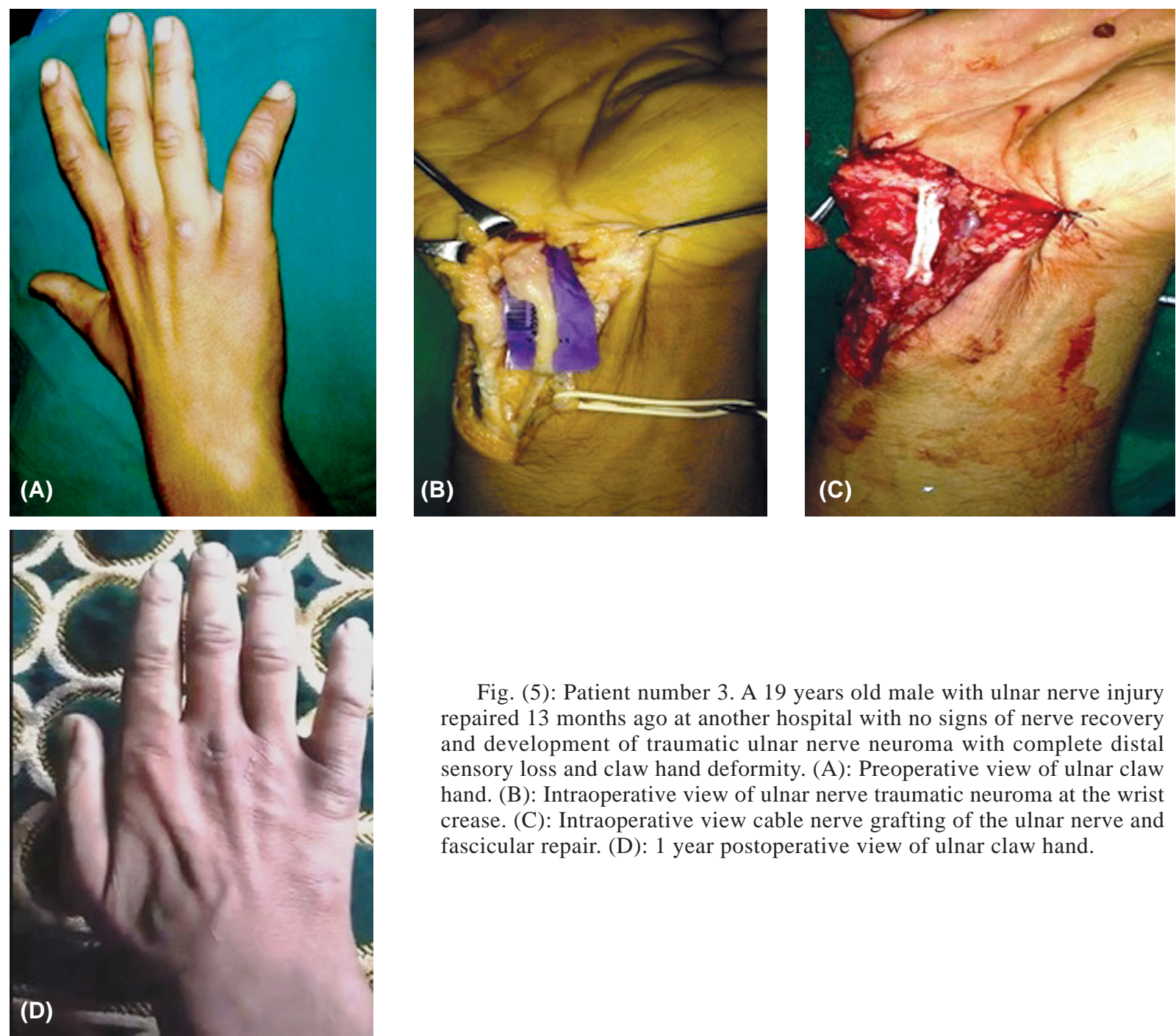

Fig. (5): Patient number 3. A 19 years old male with ulnar nerve injury repaired 13 months ago at another hospital with no signs of nerve recovery and development of traumatic ulnar nerve neuroma with complete distal sensory loss and claw hand deformity. (A): Preoperative view of ulnar claw hand. (B): Intraoperative view of ulnar nerve traumatic neuroma at the wris crease. (C): Intraoperative view cable nerve grafting of the ulnar nerve and fascicular repair. (D): 1 year postoperative view of ulnar claw hand.

\section{DISCUSSION}

In this study, we used sural nerve grafts in reconstruction of post-traumatic non-conducting ulnar nerve neuroma. Patients were relieved from pain at the site of neuroma with improvement of both sensory and motor functions.
Ulnar nerve injuries can result in poor hand functions which require multiple surgeries with demanding technique and high cost. Prolonged periods of sick leaves and subsequent functional loss can occurs even with proper treatment. These injuries can result in added economic and social burdens to the patient and the community [18]. 
It is accepted that motor restoration of ulnar nerve functions is more important than sensory restoration of ulnar nerve functions [19]. However, useful hand functions cannot be restored when there is insensate hand as the hand becomes blind especially in cases with associated median and ulnar nerve lesions [20]. Furthermore, patients with median or ulnar nerve disorders have a disturbed sensory perception, fail to perceive ordinary stimulation experienced by others and stronger stimuli are required to interact [21].

Traumatic neuroma of the ulnar nerve is a difficult clinical condition. The patient complains from extreme discomfort, pain and tenderness at the site of the neuroma with distal motor and sensory impairment of the ulnar nerve functions [22]. The condition becomes worse when cold intolerance occurs. Patient avoid the use of his hand due to severe pain even if recovery of motor functions occurs [23].

Several techniques were used for correction of neuroma in continuity of ulnar nerve. Internal neurolysis [24], nerve wrapping by vascularized tissues [25], nerve supercharging [26] and nerve resection and reconstruction [27] are the main surgical options. The results of neurolysis are superior to nerve resection and reconstruction. This was explained by the severity of injury in nerve neurolysis was lesser than the injury in nerve resection group. In addition, neurolysis is less invasive technique than neuroma resection and reconstruction [28].

The deep branch of the ulnar nerve is supercharged by the nerve to Pronator quadratus together with fascicular nerve grafting are used in cases with high ulnar nerve injuries [29]. However, all of our cases were at the level of the distal forearm or the wrist joint and we do not use nerve supercharging.

In our patients, there were history of previous nerve repair, which was complicated by severe pain at the site of previous surgery. Electrophysiological studies confirmed the lack of ulnar nerve innervation to the hand. Surgical exploration revealed the existence of ulnar nerve neuroma. Conventional nerve grafts were used to reconstruct of the neuroma in continuity and sensory improvement were observed.

However, motor recovery was not satisfying to our patients and tendon transfers were needed to improve hand functions. Our case series is secondary cases after unsuccessful previous surgical repairs. Despite nerve regeneration, the condition of the intrinsic hand muscles were poor to recover useful hand functions.

\section{Conclusion:}

Unsuccessful repair of ulnar nerve injuries can result in formation of traumatic neuroma which is a devastating clinical condition associated with poor hand function. The use of sural nerve grafts in reconstruction of traumatic non-conducting ulnar nerve neuroma resulted in pain relieve at the site of neuroma, improved sensory functions and some improvement in the motor functions.

\section{REFERENCES}

1- Sunderland S.: The anatomy and physiology of nerve injury. Muscle Nerve, 13: 771-784, 1990.

2- Mackinnon S.E. and Dellon A.L.: Results of treatment of recurrent dorsoradial wrist neuromas. Ann. Plast. Surg., 19 (1): 54-61, 1987.

3- Mackinnon S.E., Dellon A.L., Hudson A.R. and Hunter D.A.: Alteration of neuroma formation by manipulation of its microenvironment. Plast. Reconstr. Surg., 76: 345$352,1985$.

4- Yüksel F., Kişlaoğlu E., Durak N., Uçar C. and Karacaoglu E.: Prevention of painful neuromas by epineural ligatures, flaps and grafts. Br. J. Plast. Surg., 50: 182$185,1997$.

5- Sapienza A. and Green S.: Correction of the Claw Hand. Hand Clin., 28: 53-66, 2012.

6- Kouyoumdjian J.A.: Peripheral nerve injuries: A retrospective survey of 456 cases. Muscle Nerve, 34: 785, 2006.

7- Vordemvenne T., Langer M., Ochman S., et al.: Longterm results after primary microsurgical repair of ulnar and median nerve injuries: A comparison of common score systems. Clin. Neurol. Neurosurg., 109: 263, 2007.

8- Allan C.H. and Vanderhooft E.: Functional outcomes after nerve grafting. Atlas Hand Clin., 10: 93, 2005.

9- Kim D.H., Han K., Tiel R.L., Murovic J.A. and Kline D.G.: Surgical outcomes of 654 ulnar nerve lesions. J. Neurosurg., 98: 993, 2003.

10- Andreisek G., Crook D.W., Burg D., Marincek B. and Weishaupt D.: Peripheral neuropathies of the median, radial and ulnar nerves: MR imaging features. Radiographics, 26: 1267-1287, 2006.

11- Cobb T.K., Carmichael S.W. and Cooney W.P.: Guyon's canal revisited: An anatomic study of the carpal ulnar neurovascular space. J. Hand Surg., 9: 861-869, 1996.

12- Sunderland S., Marshall R.D. and Swaney W.E.: The intraneural topography of the circumflex, musculocutaneous and obturator nerves. Brain, 32: 116-29, 1959.

13- Sunderland S.: The intraneural topography of the radial, median and ulnar nerves. Brain, 68: 243-98, 1945.

14- Williams H.B. and Jabaley M.E.: The importance of internal anatomy of the peripheral nerves to nerve repair in the forearm and hand. Hand Clin., 2: 689-707, 1986. 
15- Chow J.A., Van Beek A.L., Meyer D.L. and Johnson M.C.: Surgical significance of the motor fascicular group of the ulnar nerve in the forearm. J. Hand Surg. Am., 10: 867$72,1985$.

16- Brandt K.E. and Mackinnon S.E.: Microsurgical repair of peripheral nerves and nerve grafts. In: Aston S.J., Beasley R.W., Throne C.H.M., editors. Grabb and Smith's Plastic Surgery, $5^{\text {th }}$ edition. Philadelphia: Lippincott Williams \& Wilkins, 1997.

17- Wang Y., Sunitha M., Kevin C. and Chung K.C.: How to Measure Outcomes of Peripheral Nerve Surgery. Hand Clin., 29 (3): 349-361.

18- Bergmeister K.D., Große-Hartlage L., Daeschler S.C., Rhodius P., Böcker A., Beyersdorff M., Kern A.O., Kneser U. and Harhaus L.: Acute and long-term costs of 268 peripheral nerve injuries in the upper extremity. PLoS One, 6; 15 (4): e0229530, 2020.

19- Sunderland S.: Ulnar nerve lesions. In Nerves and Nerve Injuries. Edinburgh: Churchill Livingstone, 750-779, 1978.

20- Dellon A.L., Curtis R.M. and Edgerton M.T.: Reeducation of sensation in the hand after injury and repair. Plast. Reconstr. Surg., 53: 297, 1974.

21 - Vikström P., Björkman A., Carlsson I.K., Olsson A.K. and Rosén B.: Atypical sensory processing pattern following median or ulnar nerve injury - a case-control study. BMC Neurol., Sep. 19; 18 (1): 146, 2018.

22- Watson J., Gonzalez M., Romero A. and Kerns J.: Neuromas of the Hand and Upper Extremity. J. Hand Surg., 35A: 499-510, 2010 .
23- Carlsson I., Cederlund R., Hoglund P., Lundborg G. and Rosen B.: Hand injuries and cold sensitivity: Reliability and validity of cold sensitivity uestionnaires. Disability and rehabilitation, 30: 1920-8, 2008.

24- Elliot D., Lloyd M., Hazari A., et al.: Relief of the pain of neuromas-in-continuity and scarred median and ulnar nerves in the distal forearm and wrist by neurolysis, wrapping in vascularized forearm fascial flaps and adjunctive procedures. J. Hand Surg. Eur., 35: 575-82, 2010 .

25- Jones N.F., Shaw W.W., Katz R.G., et al.: Circumferential wrapping of a flap around a scarred peripheral nerve for salvage of end-stage traction neuritis. J. Hand Surg. Am., 22: 527-35, 1997.

26- Ghoraba S.M., Mahmoud W.H., Elsergany M.A. and Ayad H.M.: Ulnar Nerve Injuries (Sunderland Grade V): A Simplified Classification System and Treatment Algorithm. Plast. Reconstr. Surg. Glob Open, 21; 7 (11): e2474, 2019.

27- Barrios C., Amillo S., Pablos J. and Canadell J.: Secondary repair of ulnar nerve injury: 44 cases followed for 2 years. Acta. Orthop. Scand, 61: 46, 1990.

28- Terzis J.K. and Kokkalis Z.T.: Outcomes of Secondary Reconstruction of Ulnar Nerve Lesions: Our Experience. Plast. Reconstr. Surg., 122: 1100, 2008.

29- Ding W., Li X., Pan J., Zhang P., Yin S., Zhou X., Li J., Wang L., Wang X. and Dong J.: Repair Method for Complete High Ulnar Nerve Injury Based on Nerve Magnified Regeneration. Ther. Clin. Risk Manag., Mar. 3 (16): 155168,2020 